\title{
Treatment effect of Bushen Huayu extract on postmenopausal osteoporosis in vivo
}

\author{
LU OUYANG ${ }^{1,2}$, QIUFANG ZHANG ${ }^{1,2,3}$, XUZHI RUAN ${ }^{3}$, YIBIN FENG $^{4}$ and XUANBIN WANG ${ }^{1,2}$ \\ ${ }^{1}$ Laboratory of Chinese Herbal Pharmacology, Renmin Hospital, Hubei University of Medicine, Shiyan, Hubei 442000; \\ ${ }^{2}$ School of Pharmacy; ${ }^{3}$ Basic School of Medicine, Hubei University of Medicine, Shiyan, Hubei 442000; \\ ${ }^{4}$ School of Chinese Medicine, LKS Faculty, The University of Hong Kong, Hong Kong, SAR, P.R. China
}

Received December 2, 2013; Accepted March 25, 2014

DOI: $10.3892 /$ etm.2014.1661

\begin{abstract}
Bushen Huayu extract (BSHY), a traditional Chinese medicine, has been demonstrated to treat postmenopausal osteoporosis, however, the underlying mechanism remains to be fully elucidated. The aim of the present study was to investigate the therapeutic effect of BSHY and the mechanisms underlying this effect in an in vivo postmenopausal osteoporosis animal model. A total of $1 \mathrm{~g}$ BSHY containing $7.12 \mu \mathrm{g}$ icariin was prepared. Low-dose BSHY (BSHY-L; $11.1 \mathrm{~g} / \mathrm{kg}$ ), medium-dose BSHY (BSHY-M; $22.2 \mathrm{~g} / \mathrm{kg}$ ) and high-dose BSHY (BSHY-H; $44.4 \mathrm{~g} / \mathrm{kg}$ ) was administered to oophorectomized rats using intragastric infusion. Estradiol (E2), interleukin-6 (IL-6) and serum alkaline phosphatase (ALP) levels, as well as bone density, were determined. It was found that the levels of serum ALP in the BSHY-L, BSHY-M and BSHY-H groups $(197.75 \pm 41.74,166.63 \pm 44.83$ and $165.63 \pm 44.90 \mathrm{IU} / 1$, respectively) were significantly decreased compared with the model group (299.13 $\pm 45.79 \mathrm{IU} / \mathrm{l}$; $\mathrm{P}<0.05)$, whilst the levels of E2 $(16.89 \pm 1.71,17.95 \pm 1.40$ and $18.34 \pm 1.43 \mathrm{pg} / \mathrm{ml}$, respectively) increased compared with the model group $(14.54 \pm 1.61 ; \mathrm{P}<0.05)$. In addition, the levels of IL-6 decreased in the BSHY-L, BSHY-M and BSHY-H groups $(91.85 \pm 14.81,82.99 \pm 15.65$ and $80.54 \pm 14.61 \mathrm{pg} / \mathrm{ml}$, respectively) compared with the model group $(105.93 \pm 16.50 \mathrm{pg} / \mathrm{ml} ; \mathrm{P}<0.05)$. Furthermore, it was demonstrated that BSHY increased the bone density in the BSHY-L, BSHY-M and BSHY-H groups $\left(0.20 \pm 0.014,0.22 \pm 0.016\right.$ and $0.22 \pm 0.017 \mathrm{~g} / \mathrm{cm}^{2}$, respectively) compared with the model group $\left(0.19 \pm 0.011 \mathrm{~g} / \mathrm{cm}^{2} ; \mathrm{P}<0.05\right)$. BSHY was also found to increase the number of osteoblasts in the BSHY-L, BSHY-M and BSHY-H groups (25.38 \pm 2.17 , $29.25 \pm 2.12$ and $30.00 \pm 2.39$, respectively), compared with in the
\end{abstract}

Correspondence to: Professor Xuanbin Wang, Laboratory of Chinese Herbal Pharmacology, Renmin Hospital, Hubei University of Medicine, 39 Chaoyang Mid-Road, Shiyan, Hubei 442000, P.R. China

E-mail: xbwang2013@163.com

Key words: traditional Chinese medicine, nilestriol, interleukin-6, alkaline phosphatase, estradiol model group $(14.75 \pm 2.38 ; \mathrm{P}<0.05)$, and decrease the number of osteoclasts in the BSHY-L, BSHY-M and BSHY-H groups $(4.00 \pm 1.85,4.25 \pm 1.39$ and $5.75 \pm 1.49$, respectively) compared with $9.50 \pm 1.60$ observed in the model group $(\mathrm{P}<0.05)$. These results suggest that $\mathrm{BSHY}$ is a potential therapeutic drug for the treatment of osteoporosis in vivo. Furthermore, these results suggest that the mechanism by which BSHY decreases the serum levels of IL-6 may be by regulating E2.

\section{Introduction}

Postmenopausal osteoporosis is a type of systemic bone disease, characterized by a reduction in bone density, degradation of bone microstructure and an alteration in serum markers of bone metabolism, including alkaline phosphatase (ALP), estradiol (E2) and interleukin-6 (IL-6) in postmenopausal females. This results in bone fragility and an increased risk of fracture. Approximately a third of postmenopausal females suffer from osteoporosis and this is usually due to the marked reduction in estrogen levels (1). Hormone replacement therapy (HRT) has been demonstrated to prevent bone loss and commonly includes a combination of estrogen, progesterone and progestin (2). However, long-term HRT results in adverse side effects, including hypocalcemia, worsening of renal impairment and osteonecrosis of the jaw (3), and therefore novel therapeutic strategies for the treatment of postmenopausal osteoporosis are required. Bushen Huауu (BSHY) is used in traditional Chinese medicine. It is based on the unique philosophy of Chinese medicine, namely Bu Shen Hua Yu (complementing the kidney system and resolving blood stasis). According to the theory of traditional Chinese medicine, BSHY is able to improve the function of the kidney system, strengthen bones, improve blood circulation and relieve pain (4). The aim of the present study was to investigate the therapeutic effects of BSHY on bone density and morphology, as well as on serum markers of bone metabolism in a postmenopausal osteoporosis animal model, and to investigate the underlying mechanisms.

\section{Materials and methods}

Drugs and reagents. All Chinese medicinal materials used in the present study were purchased from Hubei Shennong Bencao Prepared Herbal Medicines Co., Ltd. (Xuchang, 
Henan, China) and were identified by our laboratory. The herbarium specimens (no. SYRM-20090818-0090833) were deposited in Hubei University of Medicine (Shiyan, Hubei, China). The components were concentrated by decocting the solution twice in a 10 -fold volume of water at $100^{\circ} \mathrm{C}$ for $30 \mathrm{~min}$. It was found that $1 \mathrm{~g}$ BSHY corresponded with $1.2 \mathrm{~g}$ raw medicinal material. The concentration of icariin, a quantity reference compound in the extract, was $7.12 \mu \mathrm{g} / \mathrm{g}$ in a previous study (5). Nilestriol tablets were purchased from Shanghai Hualian Pharmaceutical Co., Ltd. (Shanghai, China; no. 970710$)$. Chloral hydrate (10\%) was freshly prepared in the laboratory. The E2 kit was purchased from Shenzhen Laerwen Bioengineering Technology Co., Ltd. (Shenzhen, Guangdong, China) and the IL-6 kit was purchased from Tianjin Jiuding Medical Bioengineering Co., Ltd. (Tianjin, China).

Experimental animals. A total of 48 healthy, female Sprague-Dawley rats of SPF grade, aged 3 months and weighing $215 \pm 45 \mathrm{~g}$ were provided by the Hubei Laboratory Animal Center (Wuhan, Hubei, China; License no. SCXK-E2005-0008). The rats were randomly divided into six groups: the control group, the model group, the nilestriol group, the low-dose BSHY (BSHY-L) group, the medium-dose BSHY (BSHY-M) group and the high-dose BSHY (BSHY-H) group. All experiments were conducted in accordance with the Guidelines for Ethical Conduct in the Care and Use of Experimental Animals of the Hubei University of Medicine and were approved by the ethics committee of the university.

Oophorectomy model establishment. The animal models were established as previously described with minor modifications (6). In the control group, sham surgery was performed and only a small piece of fat tissue near the ovary was removed. In all other groups, the ovaries were removed. Two weeks after the surgery, 11.1, 22.2 and $44.4 \mathrm{~g} / \mathrm{kg}$ BSHY was administered by intragastric infusion to rats in the BSHY-L, BSHY-M and BSHY-H treatment groups, respectively, whilst nilestriol $(0.1 \mathrm{mg} / \mathrm{kg})$ was administered to rats in the nilestriol group. The animals in the control and model groups underwent intragastric infusions of $10 \mathrm{ml} / \mathrm{kg}$ of $0.9 \%$ sodium chloride (equal volume to the other groups). Treatment was performed for 12 weeks and was completed $24 \mathrm{~h}$ prior to sample collection.

Sampling and detection. Following the drug treatment, blood and serum samples were collected from all the rats. Serum ALP, E2 and IL-6 levels were determined. The rats were sacrificed by cervical dislocation and the left and right femurs were collected. Dual energy X-ray absorptiometry was used to measure the bone mineral density in the right femur. Serial $4-\mu \mathrm{m}$ sections were cut at the proximal end of the left femur and the slices were stained using hematoxylin and eosin. The slices were examined using microscopy (Olympus BX43, Olympus Co., Tokyo, Japan) and the average number of osteoblasts and osteoclasts was determined.

Statistical analysis. All data are expressed as the mean \pm standard deviation. The SPSS 13.0 software package was used for statistical analysis (SPSS, Inc., Chicago, IL,

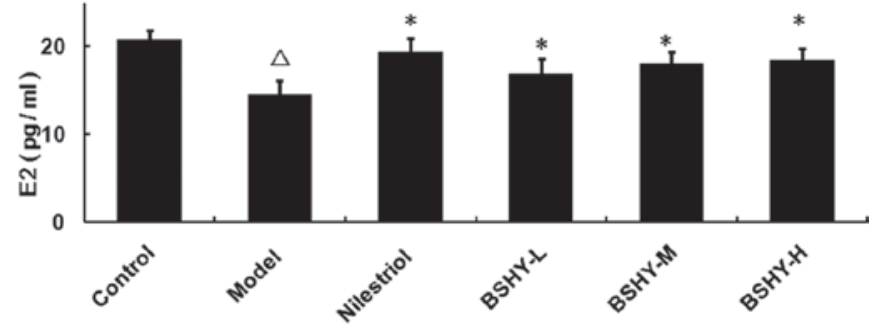

Figure 1. Effect of BSHY on serum E2 levels in rats. ${ }^{\Delta} \mathrm{P}<0.05$, vs. the control group; ${ }^{*} \mathrm{P}<0.05$, vs. the model group. BSHY, Bushen Huayu extract; E2, estradiol; BSHY-L, low-dose BSHY; BSHY-M, medium-dose BSHY; BSHY-H, high-dose BSHY

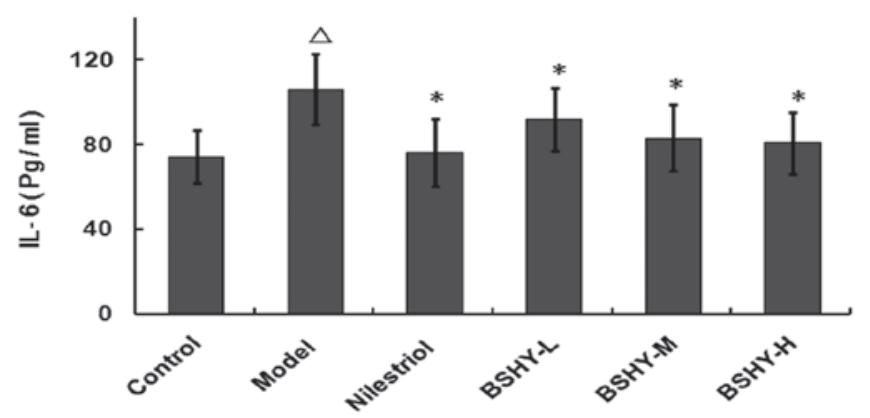

Figure 2. Effect of BSHY on serum IL-6 levels in rats. ${ }^{\Delta} \mathrm{P}<0.05$, vs. the control group; ${ }^{\mathrm{P}}<0.05$, vs. the model group. BSHY, Bushen Huауи extract; IL-6, interleukin-6; BSHY-L, low-dose BSHY; BSHY-M, medium-dose BSHY; BSHY-H, high-dose BSHY.

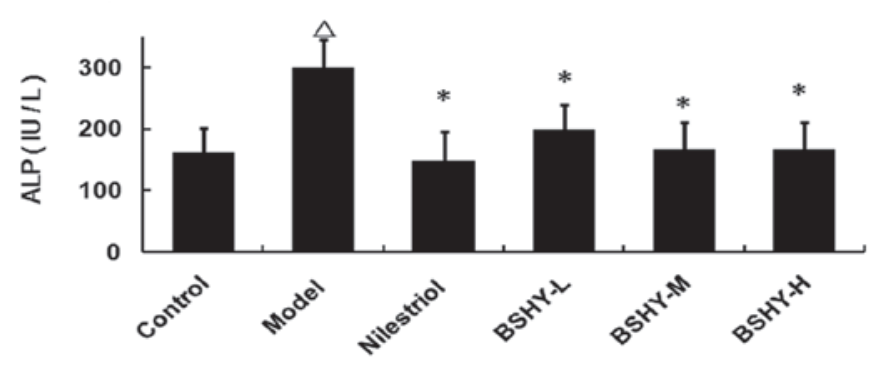

Figure 3. Effect of BSHY on serum ALP levels in rats. ${ }^{\Delta} \mathrm{P}<0.05$, vs. the control group; " $\mathrm{P}<0.05$, vs. the model group. BSHY, Bushen Huауи extract; ALP, alkaline phosphatase; BSHY-L, low-dose BSHY; BSHY-M, medium-dose BSHY; BSHY-H, high-dose BSHY.

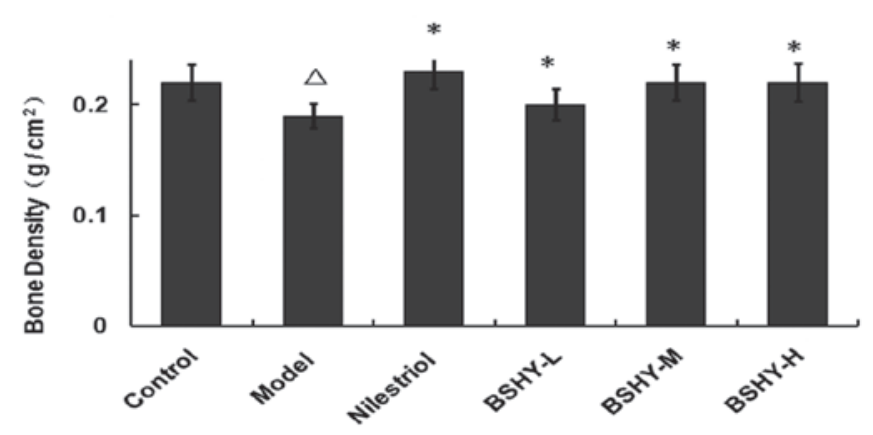

Figure 4. Effect of BSHY on bone density in the bone metaphysis of the rat right femur. ${ }^{\Delta} \mathrm{P}<0.05$, vs. the control group; ${ }^{*} \mathrm{P}<0.05$, vs. the model group. BSHY, Bushen Ниауи extract. BSHY-L, low-dose BSHY; BSHY-M, medium-dose BSHY; BSHY-H, high-dose BSHY. 

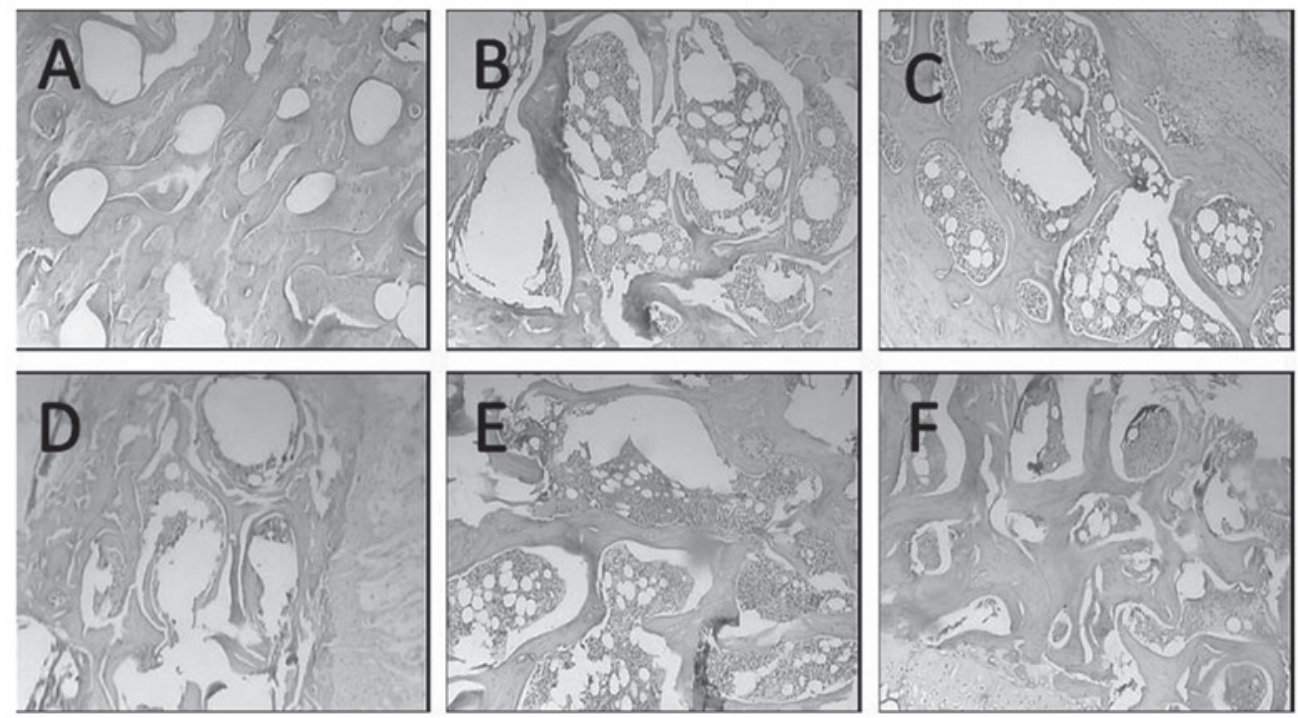

Figure 5. Effects of BSHY on bone tissue morphology in oophorectomized rats. (A) Control group; (B) model group; (C) nilestriol group; (D) low-dose BSHY group; (E) medium-dose BSHY group and (F) high-dose BSHY group. BSHY, Bushen Huayu extract.

USA). Statistical differences were evaluated using one way analysis of variance. $\mathrm{P}<0.05$ was considered to indicate a statistically significant difference.

\section{Results}

Effect of BSHY on the serum levels of E2 in oophorectomized rats. The serum E2 levels in the normal, model, nilestriol, BSHY-L, BSHY-M and BSHY-H groups were $20.71 \pm 1.08,14.54 \pm 1.61,19.34 \pm 1.59,16.89 \pm 1.71,17.95 \pm 1.40$ and $18.34 \pm 1.43 \mathrm{pg} / \mathrm{ml}$, respectively. The serum levels of E2 in the model group were significantly lower compared with the control group $(\mathrm{P}<0.05)$. The levels of E2 in the nilestriol group were significantly higher compared with the model group $(\mathrm{P}<0.05)$. Furthermore, compared with the model group, the serum levels of E2 in all three BSHY groups were significantly elevated $(\mathrm{P}<0.05)$, however, the levels remained lower than the control group (Fig. 1).

Effect of BSHY on serum IL-6 levels in oophorectomized rats. The serum levels of IL-6 in the normal, model, nilestriol, BSHY-L, BSHY-M and BSHY-H groups were $74.2 \pm 12.48$, $105.93 \pm 16.50,76.08 \pm 15.79,91.85 \pm 14.81,82.99 \pm 15.65$ and $80.54 \pm 14.61 \mathrm{pg} / \mathrm{ml}$, respectively. Compared with the control group, the serum levels of IL-6 in the model group were significantly increased $(\mathrm{P}<0.05)$, while the serum IL-6 levels in the BSHY-L, BSHY-M and BSHY-H groups were significantly lower compared with the model group ( $\mathrm{P}<0.05$; Fig. 2$)$.

Effect of BSHY on the serum levels of ALP in oophorectomized rats. The serum ALP levels in rats in the normal, model, nilestriol, BSHY-L, BSHY-M and BSHY-H groups were $159.88 \pm 40.44,299.13 \pm 45.79,147.88 \pm 48.14,197.75 \pm 41.74$, $166.63 \pm 44.83$ and $165.63 \pm 44.90 \mathrm{IU} / 1$, respectively. Compared with the control group, the serum ALP levels of rats in the model group were significantly elevated $(\mathrm{P}<0.05)$, while the levels of ALP in the three BSHY treatment groups were significantly lower compared with the model group $(\mathrm{P}<0.05)$.
However, no significant differences between the three BSHY groups and the nilestriol group were identified $(\mathrm{P}>0.05$; Fig. 3).

Effect of BSHY on bone density in the bone metaphysis of the rat right femur. The bone density in the metaphysis of the right femur in the normal, model, nilestriol, BSHY-L, BSHY-M and BSHY-H groups were $0.22 \pm 0.016,0.19 \pm 0.011,0.23 \pm 0.016$, $0.20 \pm 0.014,0.22 \pm 0.016$ and $0.22 \pm 0.017 \mathrm{~g} / \mathrm{cm}^{2}$, respectively. The bone density in the BSHY-L, BSHY-M and BSHY-H groups was elevated compared with the model group $(\mathrm{P}<0.05)$ and no significant differences between the BSHY treatment groups were observed ( $\mathrm{P}>0.05$; Fig. 4 ).

Alterations in bone tissue morphology in the proximal end of the left femurs of oophorectomized rats. The bone trabeculae in the model group were narrower, more lightly stained and more damaged arch-shaped connections were observed compared with the control group. Bone trabeculae fragments were also observed and the number was significantly reduced compared with the control group. In addition, the medullary cavity was enlarged in the model group and the quantity of bone marrow was increased, compared with the control group. However, compared with the model group, the bone trabeculae in the three BSHY groups and the nilestriol group were all wider, and the space and connections were markedly increased. Furthermore, the medullary cavity was reduced in size (Fig. 5).

\section{Discussion}

Postmenopausal osteoporosis is a systematic imbalance, in which the speed of bone resorption is greater than that of bone formation. This disease is caused by estrogen deficiency and results in microarchitectural changes, particularly bone remodeling. Certain critical molecules co-ordinate the actions of osteoblasts and osteoclasts during bone remodeling (7). As a result of estrogen deficiency during bone remodeling osteo- 
blasts release receptor activator of $N F-\kappa B$ ligand (RANKL), a member of the tumor necrosis factor (TNF) family. RANK binds to RANKL on osteoclasts, leading to the differentiation, proliferation, multinucleation, activation and survival of osteoclasts (8). In addition, osteoblasts release markers, including TNF- $\alpha$ and IL-6 (9). IL-6 generation is attenuated by sex hormones (10-12). IL- 6 has been demonstrated to directly increase the viability of osteoclasts, inhibit their apoptosis and increase the length of their life cycle, resulting in osteoporosis $(13,14)$. In the present study, BSHY-treated rats exhibited significantly elevated levels of E2, whilst levels of IL- 6 were significantly downregulated, indicating that BSHY may attenuate the alterations induced in oophorectomized rats.

By contrast, ALP is a biomarker for osteoblasts and the levels of ALP have been previously demonstrated to increase when the level of estrogen increases in osteoblastic cells in vitro (9). However, in the present in vivo study, the serum levels of ALP in rats treated with BSHY from the three groups decreased $(\mathrm{P}<0.05)$. This may be due to the fact that half of all ALP is formed in the liver and they may cross-react in the bone ALP assay. This result is consistent with a previous study (15).

BSHY, a Chinese medicinal formulation, has been proposed to supplement the kidney system and resolve blood stasis. Among its components, Herba Epimedii (termed Yinyanghuo in Chinese) and the active ingredient icariin, may have a potential role in the prevention and treatment of osteoporosis by increasing the mRNA expression levels of osteoprotegerin (a TNF-related cytokine), bone morphogenetic protein (a promoter of osteogenesis) and collagen I (synthesized by active osteoblasts) in oophorectomized rats (16), as well as inducing estrogen biosynthesis (17). Rhizoma Drynariae (termed Gusuibu in Chinese) may enhance the treatment effects on osteoporosis by reducing metabolic disorder (18). The anti-osteoporosis mechanisms of other traditional Chinese medicines remain to be elucidated. They may have a combined synergistic effect with Herba Epimedii and Rhizoma Drynariae to enhance the actions and/or reduce the side-effects.

Further studies are required to investigate whether other bone resorption and bone formation parameters, including RANKL, RANK, tartrate-resistant acid phosphatase, IL-1, calcium, osteocalcin, TNF- $\alpha$ and deoxypyridinoline, are also involved in BSHY treatment in oophorectomized rats.

In conclusion, in the present study, BSHY extract was demonstrated to have a therapeutic effect on osteoporosis caused by oophorectomy, and thus may be a potential therapeutic treatment for postmenopausal osteoporosis. Furthermore, these results suggest that the mechanism by which BSHY decreases the serum levels of IL-6 may be by regulating $\mathrm{E} 2$.

\section{Acknowledgements}

This study was supported by the Class B Funding of the Education Department of Hubei Province, China (Code no. B20082412), The Public Health Project of Shiyan City (grant no. 2011057) and Hubei 2011 Research Project Funding (grant no. 4). The authors would like to thank Mr. Hongliang Li, Mrs. Ming Liu and Mrs. Hongli Guo for their technical support.

\section{References}

1. Cavalli L and Brandi ML: Age- and gender-related macro- and micro-architecture changes in bone structure and implications for treatment. Int J Clin Rheumatol 6: 359-369, 2011.

2. Lindsay R: Hormones and bone health in postmenopausal women. Endocrine 24: 223-230, 2004.

3. Sanders S and Geraci SA: Osteoporosis in postmenopausal women: considerations in prevention and treatment: (women's health series). South Med J 106: 698-706, 2013.

4. Yoldemir T, Erenus M and Durmusoglu F: The impact of serum FSH and estradiol on postmenopausal osteoporosis related to time since menopause. Gynecol Endocrinol 28: 884-888, 2012.

5. Ouyang L, Wang X, Li H and Xiao Y: Determination of Icariin in Bushenhuayu Electuary by HPLC. Zhong Guo Yao Shi 13: 13-15, 2010 (In Chinese).

6. Joo MK, Park JJ, Lee BJ, Kim JH, Yeon JE, Kim JS, Byun KS and Bak YT: The effect of a proton pump inhibitor on bone metabolism in ovariectomized rats. Mol Med Rep 7: 1267-1272, 2013.

7. Rachner TD, Khosla S and Hofbauer LC: Osteoporosis: now and the future. Lancet 377: 1276-1287, 2011.

8. Craft CS, Broekelmann TJ, Zou W, Chappel JC, Teitelbaum SL and Mecham RP: Oophorectomy-induced bone loss is attenuated in MAGP1-deficient mice. J Cell Biochem 113: 93-99, 2012.

9. Kwak EJ,Lee YS and Choi EM: Effect of magnolol on the function of osteoblastic MC3T3-E1 cells. Mediators Inflamm 2012: 829650, 2012.

10. Wang M, Ling G, Bei X, Junqing C, PeiqingZ and Jie H: Clinical observation on 96 cases of primary osteoporosis treated with kidney-tonifying and bone-strengthening mixture. J Tradit Chin Med 25: 132-136, 2005.

11. Hsieh TP, Sheu SY, Sun JS and Chen MH: Icariin inhibits osteoclast differentiation and bone resorption by suppression of MAPKs/NF- $\kappa$ B regulated HIF-1 $\alpha$ and PGE(2) synthesis. Phytomedicine 18: 176-185, 2011.

12. McLean RR: Proinflammatory cytokines and osteoporosis. Curr Osteoporos Rep 7: 134-139, 2009.

13. Mysliwiec J, Adamczyk M, Nikolajuk A and Gorska M: Interleukin-6 and its considerable role in the pathogenesis of thyrotoxicosis-related disturbances of bone turnover in postmenopausal women. Endokrynol Pol 62: 299-302, 2011.

14. Wang Y, Li LZ, Zhang YL, Zhu YQ, Wu J and Sun WJ: LC, a novel estrone-rhein hybrid compound, concurrently stimulates osteoprotegerin and inhibits receptor activator of $\mathrm{NF}-\mathrm{\kappa B}$ ligand (RANKL) and interleukin-6 production by human osteoblastic cells. Mol Cell Endocrinol 337: 43-51, 2011.

15. Eastell R, Reid DM, Vukicevic S, Ensrud KE, LaCroix AZ, Thompson JR, Thompson DD and Cummings SR: Effects of 3 years of lasofoxifene treatment on bone turnover markers in women with postmenopausal osteoporosis. Bone 50: 1135-1140, 2012.

16. Song Y, Li SH, He ZJ, Zhang YW and Zhou MW: Effect of icariin on osteoporosis in ovariectomized female rats. Jun Yi Jin Xiu Xue Yuan Xue Bao 33: 400-403, 2012 (In Chinese).

17. Yang L, Lu D, Guo J, Meng X, Zhang G and Wang F: Icariin from Epimedium brevicornum Maxim promotes the biosynthesis of estrogen by aromatase (CYP19). J Ethnopharmacol 145: 715-721, 2012 .

18. Zhang S, Liu X, Zheng S, Jiang M, Xin C, Lu X, Li F and Xiong Z: Metabonomic study on protective effect of ethanol extracts of drynariae rhizoma on osteoporosis in rats urine by using UPLC-MS/MS. Zhongguo Zhong Yao Za Zhi 37: 658-662, 2012 (In Chinese). 